\title{
Stability Analysis of Clamped Nonhomogeneous Shells on the Elastic Foundation
}

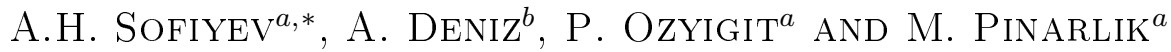 \\ ${ }^{a}$ Department of Civil Engineering, Suleyman Demirel University, Isparta, Turkey \\ ${ }^{b}$ Department of Mathematics, Usak University, Usak, Turkey
}

\begin{abstract}
In the present study a theoretical analysis is presented for determining the stability characteristics of clamped non-homogeneous shells on the elastic foundation subjected to the lateral pressure. The basic equations have been derived for the shell, the Young modulus of which varies exponentially in the thickness direction and rests on the elastic foundation. By applying the Galerkin method to the basic equations, the expressions for the critical lateral pressure of the non-homogeneous shell with or without an elastic foundation are obtained. Finally, the effects of the non-homogeneity, elastic foundation and shell characteristics on the critical lateral pressure have been studied.

DOI: $10.12693 /$ APhysPolA.125.459

PACS: 61.43.Bn, 62.20.mq
\end{abstract}

\section{Introduction}

In many practical applications, particularly in aerospace industry, modern missile technology, pressure vessels, water ducts, pipelines and casing pipes, shell-type structural elements have to work under radiation and high temperature environment which causes non-homogeneity in the material, i.e. mechanical properties of the material vary with space coordinates [1]. Non-homogeneous materials are of considerable interest to design engineers in various technological situations. Thus, their design requires an accurate analysis for their stability characteristics.

Up till now, several studies have been devoted to the stability and vibration behaviors of homogeneous and non-homogeneous structural elements with different boundary conditions [2-4]. The static and dynamic interaction of shells with elastic media is a problem of current importance. However, the static and dynamic analyses of clamped shells surrounded by elastic media are quite limited $[5,6]$.

In the present study a theoretical analysis is presented for determining the stability characteristics of clamped non-homogeneous shells on the elastic foundation subjected to the lateral pressure. The basic equations have been derived for the shell, the Young modulus of which varies exponentially in the thickness direction and rests on the elastic foundation. By applying the Galerkin method to basic equations, the expressions for the critical lateral pressure of the non-homogeneous shell with or without an elastic foundation are obtained. Finally, the effects of non-homogeneity, elastic foundation and shell characteristics on the critical lateral pressure have been studied.

*corresponding author; e-mail: as of iyev@mmf . sdu.edu.tr

\section{Basic equations}

The geometry of the non-homogeneous truncated conical shell is defined in Fig. 1. $R_{1}$ and $R_{2}$ are the average radii of the cone at its small and large edges, $h$ is the cone thickness, $\gamma$ is the semi-vertex angle of the cone and $L$ is the truncated cone length along its generator. The conical shell is referred to orthogonal curvilinear coordinates $(S, \theta, z)$ as shown in Fig. 1, in which $S$ is in the generatrix direction measured from the vertex of the shell, $\theta$ is in the circumferential direction, and $z$ is in the normal direction measured from the reference surface, positive inward. $S_{1}$ and $S_{2}$ being the coordinates of the points where this axis intersects the small and large bases, respectively. The Young modulus of the shell varies exponentially in the thickness direction as $E(\bar{z})=E_{0}\left(1+\mu \mathrm{e}^{-0.1|\bar{z}|}\right)$ and Poisson's ratio is assumed to be constant $[4,5]$. Here $\bar{z}=z / h$, $E_{0}$ is the Young modulus of the homogeneous isotropic material and $\mu$ is the non-homogeneity coefficient, satisfying $0 \leq \mu<1$. The non-homogeneous truncated conical shell rests on the Winkler elastic foundation. The load-displacement relationship of the foundation is assumed to be $N=K w$, where $N$ is the force per unit area, $K$ is the Winkler foundation stiffness and $w$ is the displacement in the radial direction.

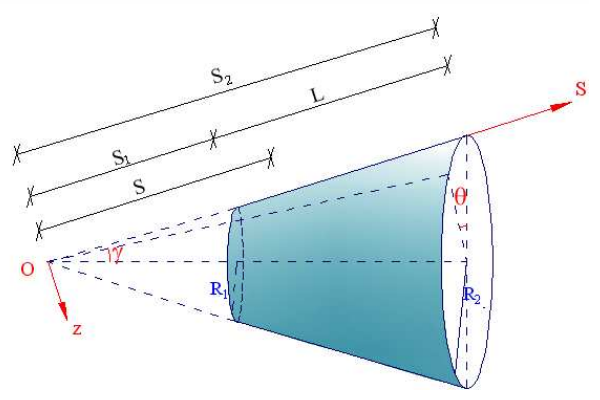

Fig. 1. Geometry of a truncated conical shell. 
Considering the above mentioned knowledge, the stability and compatibility equations of a non-homogeneous isotropic truncated conical shell subjected to the uniform lateral pressure and resting on the Winkler elastic foundation can be written in the following form as:

$$
\begin{aligned}
& \mathrm{e}^{-4 x}\left[\delta_{1} \frac{\partial^{4} \Psi}{\partial x^{4}}-4 \delta_{1} \frac{\partial^{3} \Psi}{\partial x^{3}}+4 \delta_{1} \frac{\partial^{2} \Psi}{\partial x^{2}}+\delta_{1} \frac{\partial^{4} \Psi}{\partial \varphi^{4}}\right. \\
& +\delta_{2} \frac{\partial^{4} \Psi}{\partial x^{2} \partial \varphi^{2}}-2 \delta_{2} \frac{\partial^{3} \Psi}{\partial x \partial \varphi^{2}}+\left(\delta_{2}+2 \delta_{1}\right) \frac{\partial^{2} \Psi}{\partial \varphi^{2}} \\
& -\delta_{3} \frac{\partial^{4} w}{\partial \varphi^{4}}-\delta_{4} \frac{\partial^{4} w}{\partial x^{2} \partial \varphi^{2}}+2 \delta_{4} \frac{\partial^{3} w}{\partial x \partial \varphi^{2}} \\
& \left.-\left(\delta_{4}+2 \delta_{3}\right) \frac{\partial^{2} w}{\partial \varphi^{2}}-\delta_{3} \frac{\partial^{4} w}{\partial x^{4}}+4 \delta_{3} \frac{\partial^{3} w}{\partial x^{3}}-4 \delta_{3} \frac{\partial^{2} w}{\partial x^{2}}\right] \\
& +\mathrm{e}^{-3 x}\left(\frac{\partial^{2} \Psi}{\partial x^{2}}-\frac{\partial \Psi}{\partial x}\right) S_{2} \cot \gamma \\
& -S_{2}^{3} \mathrm{e}^{-x}\left(\frac{\partial^{2} w}{\partial \varphi^{2}}+\frac{\partial w}{\partial x}\right) P \tan \gamma-S_{2}^{4} K_{1} w=0 \\
& +\Delta_{1} \frac{\partial^{4} \Psi}{\partial x^{4}}-4 \Delta_{1} \frac{\partial^{3} \Psi}{\partial x^{3}}+4 \Delta_{1} \frac{\partial^{2} \Psi}{\partial x^{2}}+\Delta_{1} \frac{\partial^{4} \Psi}{\partial \varphi^{4}} \\
& +\Delta_{2} \frac{\partial^{4} \Psi}{\partial x^{2} \partial \varphi^{2}}-2 \Delta_{2} \frac{\partial^{3} \Psi}{\partial x \partial \varphi^{2}}+\left(\Delta_{2}+2 \Delta_{1}\right) \frac{\partial^{2} \Psi}{\partial \varphi^{2}} \\
& +\Delta_{3} \frac{\partial^{4} w}{\partial \varphi^{4}}+\Delta_{4} \frac{\partial^{4} w}{\partial x^{2} \partial \varphi^{2}}-2 \Delta_{4} \frac{\partial^{3} w}{\partial x \partial \varphi^{2}} \\
& +-3 x\left(\frac{\partial^{2} w}{\partial x^{2}}-\frac{\partial w}{\partial x}\right) S_{2} \cot \gamma=0 \\
& +\delta_{3}
\end{aligned}
$$

where $\delta_{i}, \Delta_{i}(i=1,2, \ldots, 4)$ are the parameters depending on the non-homogeneous material properties and shell characteristics $[3,6]$.

\section{Solution of basic equations}

Two end edges of the non-homogeneous truncated conical shells are assumed to be clamped. Thus, the approximate solution for Eqs. (1) and (2) may be assumed as $[2,3]:$

$$
\begin{aligned}
& w=A \mathrm{e}^{\lambda x} \sin ^{2}\left(m_{1} x\right) \cos \left(n_{1} \varphi\right), \\
& \Psi=B S_{2} \mathrm{e}^{(\lambda+1) x} \sin ^{2}\left(m_{1} x\right) \cos \left(n_{1} \varphi\right),
\end{aligned}
$$

where $A$ and $B$ are the amplitudes, $m_{1}=m \pi / x_{0}$, $n_{1}=n / \sin \gamma, m, n$ are wave numbers. The parameter $\lambda$ changes depending on the geometry of the shell, loading and boundary conditions, and is found from the minimum condition of the critical lateral pressure. Multiplying Eqs. (1) and (2) by $w S_{2}^{2} \mathrm{e}^{2 x} \mathrm{~d} \varphi \mathrm{d} x$ and $\Psi S_{2}^{2} \mathrm{e}^{2 x} \mathrm{~d} \varphi \mathrm{d} x$, respectively, and integrating in the ranges $0 \leq \varphi \leq$ $2 \pi \sin \gamma$ and $-x_{0} \leq x \leq 0$, i.e. applying Galerkin's method to the foregoing equations and substituting (3) into (1) and (2), the following expression for the uniform critical lateral pressure is obtained:

$$
\begin{aligned}
& P_{\mathrm{crL}}^{\mathrm{NHw}}=\left[\left(T_{1} \eta_{-1}+T_{3} \eta_{0}\right)\left(T_{6} \eta_{-1}+T_{3} \eta_{0}\right)\right. \\
& \left.\quad+T_{5} T_{2} \eta_{0} \eta_{-2}+T_{w} T_{5} \eta_{0} \eta_{2}\right] /\left(T_{5} T_{\mathrm{PL}} \eta_{0} \eta_{1}\right),
\end{aligned}
$$

where the following definitions apply:

$$
\begin{aligned}
T_{1} & =\delta_{1} U_{1}-4 \delta_{1} U_{2}+\left(4 \delta_{1}-\delta_{2} n_{1}^{2}\right) U_{3}+24 \delta_{1} n_{1}^{4} \\
& +48 \delta_{2} n_{1}^{2}-48 \delta_{1} n_{1}^{2}, \\
T_{2} & =24 \delta_{3} n_{1}^{4}-\left(\delta_{4} n_{1}^{2}-4 \delta_{3}\right) U_{4}+24 n_{1}^{2}\left(\delta_{4}-2 \delta_{3}\right) \\
& +\delta_{3} U_{5}-4 \delta_{3} U_{6}, \\
T_{3} & =-8\left(\lambda^{2}+4 m_{1}^{2}\right) S_{2} \cot \gamma, \\
T_{\mathrm{PL}} & =12\left(2 \beta_{2}^{2}+1\right) S_{2}^{3} \tan \gamma, \\
T_{5} & =24 \Delta_{1} n_{1}^{4}-\Delta_{2} n_{1}^{2} U_{7}+24 n_{1}^{2} \Delta_{2}-48 n_{1}^{2} \Delta_{1}+\Delta_{1} U_{8} \\
& -4 \Delta_{1} U_{9}+4 \Delta_{1} U_{7}, \\
T_{6} & =-24 \Delta_{3} n_{1}^{4}-\left(4 \Delta_{3}+\Delta_{4} n_{1}^{2}\right) U_{10}+48 n_{1}^{2} \Delta_{3} \\
& -\Delta_{3} U_{11}+4 \Delta_{3} U_{12}, \\
T_{w} & =24 S_{2}^{4} K, \\
\eta_{i} & =\frac{m_{1}^{4}\left[1-\mathrm{e}^{-(2 \lambda+i) x_{0}}\right]}{(2 \lambda+i)\left[(2 \lambda+i)^{2}+4 m_{1}^{2}\right]\left[(2 \lambda+i)^{2}+16 m_{1}^{2}\right]}, \\
i & =-2,-1,0,1,2,
\end{aligned}
$$

in which $U_{i}(i=1,2, \ldots, 8)$ are the parameters depending on the material properties and shell characteristics and are given in [6]. The minimum values of critical lateral pressures of homogeneous $(\mathrm{H})$ and non-homogeneous $(\mathrm{NH})$ cylindrical and truncated conical shells with and without elastic foundation are obtained by minimizing Eqs. (4) with respect to $m, n$ and $\lambda$. As $K=0$, from Eq. (4), the expression for critical lateral pressure of $\mathrm{NH}$ truncated conical shell without an elastic foundation $\left(P_{\mathrm{crL}}^{\mathrm{NH}}\right)$ is obtained. As $\gamma \rightarrow 0$, from Eq. (4), the expressions for critical lateral pressure of $\mathrm{NH}$ cylindrical shells with $\left(P_{\mathrm{crLcyl}}^{\mathrm{NHw}}\right)$ and without $\left(P_{\mathrm{crLcyl}}^{\mathrm{NH}}\right)$ elastic foundation are obtained. As $\mu=0$, from Eq. (4), the expressions for critical lateral pressure of homogeneous cylindrical and conical shells with and without an Winkler elastic foundation are obtained.

\section{Numerical computations and results}

A computer program has been used to compute numerical values from the formulae obtained. The material properties of shells are: $E_{0}=2 \times 10^{11} \mathrm{~Pa} ; \nu_{0}=0.3$. The numerical computations show that the minimum values of critical lateral pressure of clamped cylindrical and truncated conical shells on the Winkler elastic foundation are obtained approximately at $\lambda=0$ and $\lambda=4$, respectively. By taking into account above values for the parameter $\lambda$ and for the longitudinal wave number $m=1$, the critical lateral pressures are minimized only according to $n$.

The variation of critical lateral pressures for $\mathrm{H}$ and $\mathrm{NH}$ cylindrical and truncated conical shells with or without the Winkler foundation versus the semi-vertex angle, $\gamma$ and the ratio, $R_{1} / h$, for the Winkler foundation stiffness $K=10^{8} \mathrm{~N} / \mathrm{m}^{3}$, are given in Fig. $2 \mathrm{a}$ and $\mathrm{b}$, respectively. 


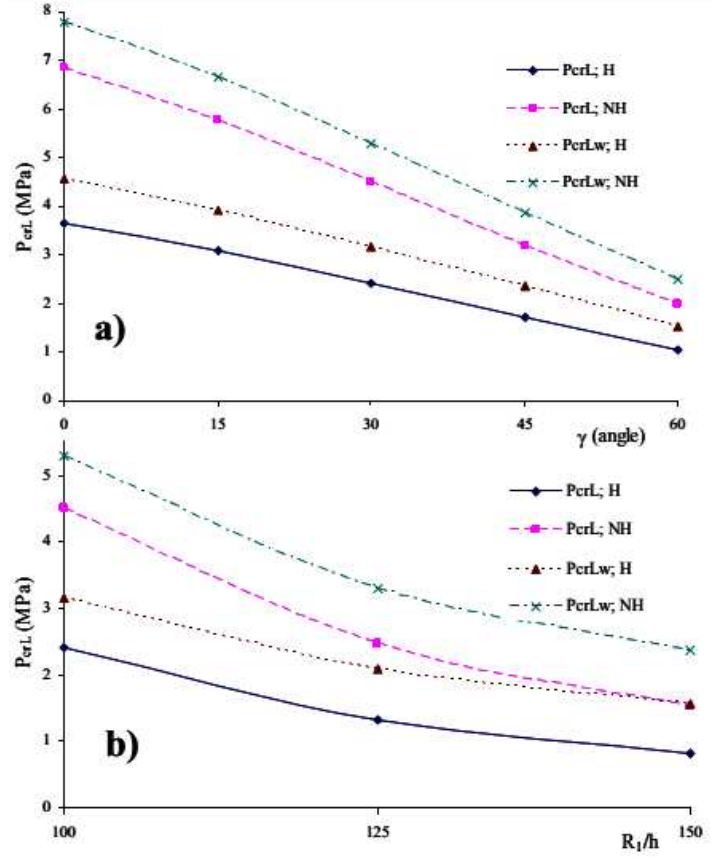

Fig. 2. Variations of critical lateral pressures of $\mathrm{H}$ and NH shells on the Winkler foundation versus (a) the semi-vertex angle, $\gamma$ and (b) the ratio, $R_{1} / h$.

The Young modulus of the shell material varies exponentially in the thickness direction and the non-homogeneity coefficient is $\mu=0.9$. As the semi-vertex angle $\gamma$ and the ratio $R_{1} / h$ increase, the values of the critical lateral pressures for $\mathrm{H}$ and $\mathrm{NH}$ truncated conical shells with or without a Winkler foundation decrease. As $\gamma$ and $R_{1} / h$ increase, the influence of the Winkler foundation on the critical lateral pressures for $\mathrm{H}$ and $\mathrm{NH}$ shells increases. The influence of the foundation on the critical lateral pressure of non-homogeneous shells is lower than the homogeneous shells. With increasing $\gamma$ and $R_{1} / h$, the influence of non-homogeneity on $P_{\mathrm{crL}}^{\mathrm{NHw}}$ is reduced from $69 \%$ to $62 \%$ and from $67 \%$ to $51 \%$, respectively, at the time, this effect on the values of $P_{\mathrm{crL}}^{\mathrm{NH}}$ remains constant $(87 \%)$. It is observed that the values of the critical lateral pressure of the cylindrical shell $(\gamma \rightarrow 0)$ is higher than the truncated conical shell, while the effect of non-homogeneity and elastic foundation on the critical lateral pressure of the cylindrical shell is smaller (see Fig. 2a).

\section{Conclusions}

In this study, the buckling of the clamped non-homogeneous shells on the elastic foundation subjected to the uniform lateral pressure has been investigated. First, the basic equations have been derived for the shell, the Young modulus of which varies exponentially in the thickness direction and rests on the elastic foundation. By applying the Galerkin method to basic equations, the expressions for the critical lateral pressure of the non-homogeneous shell with or without an elastic foundation are obtained. Finally, the influences of the non-homogeneity, elastic foundation, and shell characteristics on the values of the critical lateral pressure have been studied.

\section{Acknowledgments}

Authors thank Scientific and Technical Research Council of Turkey (TÜBİTAK) for the support of project $110 \mathrm{M} 695$.

\section{References}

[1] D.V. Babich, L.P. Khoroshun, Int. Appl. Mech. 37, 837 (2001).

[2] J. Singer, M. Baruch, J. Reichenthal, Isr. J. Tech. 9, 127 (1971)

[3] A.H. Sofiyev, N. Kuruoglu, H.M. Halilov, Appl. Math. Model. 34, 1807 (2010).

[4] R. Lal, Y. Kumar, Meccanica 47, 175 (2012).

[5] E. Bagherizadeh, Y. Kiani, M.R. Eslami, Compos. Struct. 93, 3063 (2011).

[6] A.H. Sofiyev, N. Kuruoglu, J. Eng. Math. 77, 131 (2012). 